\title{
Analysis of the genomic sequence of Philosamia cynthia nucleopolyhedrin virus and comparison with Antheraea pernyi nucleopolyhedrin virus
}

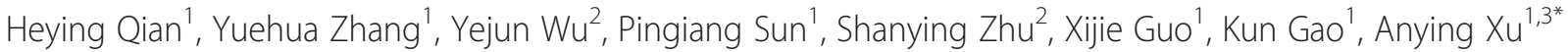 \\ and Wenbing Wang ${ }^{2}$
}

\begin{abstract}
Background: Two species of wild silkworms, the Chinese oak silkworm (Antheraea pernyi) and the castor silkworm Philosamia cynthia ricini, can acquire a serious disease caused by Nucleopolyhedrin Viruses (NPVs) (known as AnpeNPV and PhcyNPV, respectively). The two viruses have similar polyhedral morphologies and their viral fragments share high sequence similarity. However, the physical maps of the viral genomes and cross-infectivity of the viruses are different. The genome sequences of two AnpeNPV isolates have been published.

Results: We sequenced and analyzed the full-length genome of PhcyNPV to compare the gene contents of the two viruses. The genome of PhcyNPV is 125, $376 \mathrm{bp}$, with a $\mathrm{G}+\mathrm{C}$ content of 53.65\%, and encodes 138 open reading frames (ORFs) of at least 50 amino acids (aa) (GenBank accession number: JX404026). Between PhcyNPV and AnpeMNPV-L and -Z isolates, 126 ORFs are identical, including 30 baculovirus core genes. Nine ORFs were only found in PhcyNPV. Four genes, cath, $v$-chi, lef 10 and lef 11, were not found in PhcyNPV. However, most of the six genes required for infectivity via the oral route were found in PhcyNPV and in the two AnpeNPV isolates, with high sequence similarities. The pif-3 gene of PhcyNPV contained 59 aa extra amino acids at the N-terminus compared with AnpeNPV.

Conclusions: Most of the genes in PhcyNPV are similar to the two AnpeNPV isolates, including the direction of expression of the ORFs. Only a few genes were missing from PhcyNPV. These data suggest that PhcyNPV and AnpeNPV might be variants of each other, and that the differences in cross-infection might be caused by gene mutations.
\end{abstract}

\section{Background}

Baculoviridae is a large family of viruses that infect and kill insect species of different orders. Worldwide, they have been reported to infect over 600 host species [1], mostly from the order Lepidoptera. However, the viruses also infect insects from the orders Diptera, Hymenoptera and the crustacean order, Decapoda [2].

The complete genomes of 57 baculoviruses have been deposited in GenBank, including 41 Alphabaculoviruses, 12 Betabaculoviruses, three Gammabaculoviruses and one

\footnotetext{
* Correspondence: srixay@126.com

${ }^{1}$ Jiangsu University of Science and Technology, Sibaidu Road 1, Zhenjiang 212018, China

${ }^{3}$ The Sericultural Research Institute, Chinese Academy of Agricultural Sciences, Jiangsu University of Science and Technology, Sibaidu Road 1, Zhenjiang 212018, China

Full list of author information is available at the end of the article
}

Deltabaculovirus genome [3]. Baculoviruses have been used extensively in many biological applications, for example as protein expression systems, as models of genetic regulatory networks and genome evolution, as putative nonhuman viral vectors for gene delivery, and as biological control agents against insect pests [4-6]. However, the diseases caused by baculoviruses are a major threat to the silk industry [3].

Silkmoths mostly belong to two families, the Bombycidae and Saturniidae, which secrete several varieties of silk fibers. The most common breeds are the domesticated silkworm (Bombyx mori L.) and the wild silkworms, including Chinese oak silkworm (Antheraea pernyi Guérin-Meneville), the castor silkworm (Philosamia cynthia ricini), the Indian tropical tasar silkworm (A. mylitta Drury) and the Japanese oak silkworm (A. ayamamai Guérin-Meneville) [7]. Silk production 
by these moths, especially $B$. mori, A. pernyi and P. cynthia ricini, are economically important worldwide. The domesticated silkworm (B. mori) has been used for silk production by Chinese farmers for approximately 5000 years [7]. It has since spread to Korea, Japan, India, Brazil and the rest of the world. The most well-known species among wild silkworms is the Chinese oak silkworm (A. pernyi). It is commercially cultivated for silk production, primarily in China, India and Korea [8]. This silkworm species and the castor silkworm (P. Cynthia ricini) were introduced into China for silk production in the 1950s [7].

These three species can be infected by baculoviruses: B. mori Nucleopolyhedrosis virus (BmNPV), A. pernyi NPV (AnpeNPV) and P. Cynthia ricini NPV (PhcyNPV) according to their respective hosts [9-12]. The complete sequence of BmNPV strain T3 (GenBank: NC_001962) was published in 1999, and the sequence of $B$. mandarina NPV (BomaNPV, a variant of BmNPV) was published in 2010 (GenBank: FJ882854) [13,14]. Two AnpeNPV isolates were published in 2007 (GenBank: EF207986 and NC_008035). We compared AnpeNPV and PhcyNPV, and found that these two viruses have similar polyhedral morphologies and their viral fragments show high sequence similarity. However, the physical maps of the viral genomes and cross-infectivity of the viruses are different. Therefore, we analyzed the whole genome sequence of PhcyNPV to obtain more information about the viral genes related to infection.

\section{Results}

Sequencing, assembly, and analysis of the PhcyNPV genome

The entire PhcyNPV dsDNA genome was sequenced and assembled into a contiguous sequence of $125,376 \mathrm{bp}$, with a G + C content of 53.65\% (GenBank: JX404026). Many baculoviruses have an approximate GC content of $41 \%$, whereas PhcyNPV and several other baculoviruses have significantly higher values $(50.1 \%$ for CfMNPV, $50.9 \%$ for CuniNPV, 53.5\% for AnpeNPV-L2, 53.5\% for AnpeNPV-Z, $53.5 \%$ for LyxyNPV, $55.1 \%$ for OpMNPV and $57.5 \%$ for LdMNPV). However, a detailed analysis of DNA content did not show a clear pattern of GC content that could be associated with each genus [7].

One hundred and thirty-eight ORFs were identified in the PhcyNPV genome that encoded putative proteins of at least 50 amino acids (aa) with minimal adjacent ORF overlap. Designating the polyhedrin gene as the first ORF and noting its clockwise orientation, 65 ORFs were identified in the clockwise orientation, and 76 were identified in the counter-clockwise orientation. A BLAST analysis of PhcyNPV ORFs showed that 94.9\% (131/138) had assigned functions or homologs in other baculovirus genomes (Figure 1 and Additional file 1: Table S1). Seven unique ORFs were identified (nos. 18, 42, 43, 53,
92, 96, and 135), which putatively encoded polypeptides from 68 to 223 aa. However, the directions of transcription of these genes are different, and it is not known if they are expressed.

\section{Comparison of the PhcyNPV genome with the genomes of two AnpeNPV isolates}

Most genes in the PhcyNPV genome are similar to the previously published sequences of AnpeNPV, -L2 isolate (126, 246 bp, GenBank: EF207986) and -Z isolate (126, 630 bp, GenBank: NC_008035). One hundred and twenty-six ORFs are identical between PhcyNPV and the AnpeMNPV-L2 and -Z isolates, including 30 baculovirus core genes (Figure 1). Some ORFs appear to be present only in one of the isolates: nine ORFs in PhcyNPV, six in AnpeNPV-L2 and seven in AnpeNPV-Z (Figure 2). Comparing the three viral genomes, the main changes of the ORFs were found in five regions. In the region of 30 , 622 to 32, 874 nt of PhcyNPV, two genes, cath and vchi, are missing in PhcyNPV. Indels in the coding regions of five ORFS caused their lengths to vary. In region 38, 639 to 39, 754 nt, there are three ORFs (Phcy ORF41 - 43) in PhcyNPV that are not present in AnpeNPV-L2. Conversely, there are three ORFs in AnpeNPV-L2 with lengths of 82 (Anpe-ORF044), 56 (Anpe-ORF045), and 92 aa $(\operatorname{cht} B$ 2) in this region. Interestingly, in this region, AnpeNPV-Z has a homolog of PhcyNPV ORF41 (132 aa) with a length of 71 aa. Homologous genes for PhcyNPV ORF41 were also found in some other baculoviruses, such as Choristoneura fumiferana DEF MNPV (Cf DEF MNPV, orf107, 135 aa), Hyphantria cunea NPV (HycuNPV, ORF43, 135aa) and Anticarsia gemmatalis NPV (AngeNPV, AGNV_gp111, 135aa). Although Anpe-ORF044 and AnpeORF045 were found in AnpeNPV-Z, the chtB-2 gene was missing in this region. Additionally, the $p 12$ and AnpeORF069 genes were found in the PhcyNPV genome and the AnpeNPV L2 isolate, but both genes were missing in AnpeNPV-Z. In the PhcyNPV genome, lef 10 and lef 11 could not be identified.

Certain ORFS were truncated or extended. For example, the $p 94$ (Ac 134) genes in AnpeNPV-L2 and in PhcyNPV were predicted to encode proteins of 492 aa and 795 aa, respectively, whereas, in the $-\mathrm{Z}$ isolate, the gene could be divided into two ORFs (390 aa and 99 aa). Homologs of $p 94$ are present in the genomes of most Group I baculoviruses. They are also present in some members of Group II and GV, and in several polydnaviruses. Disruption of the $p 94$ gene had no effect on the ability of AcMNPV to infect $S$. frugiperda larvae by either the oral or intrahemocelic route [15]. Interestingly, the egt genes of these three viruses are different; the ORF of PhcyNPV egt comprises 87 aa in the EGT N-terminus of AcMNPV, similar to AnpeNPV (-L2, 132 aa; $-Z, 79$ aa). The egt gene encodes ecdysteroid UDP-glucosyltransferase (EGT), and 


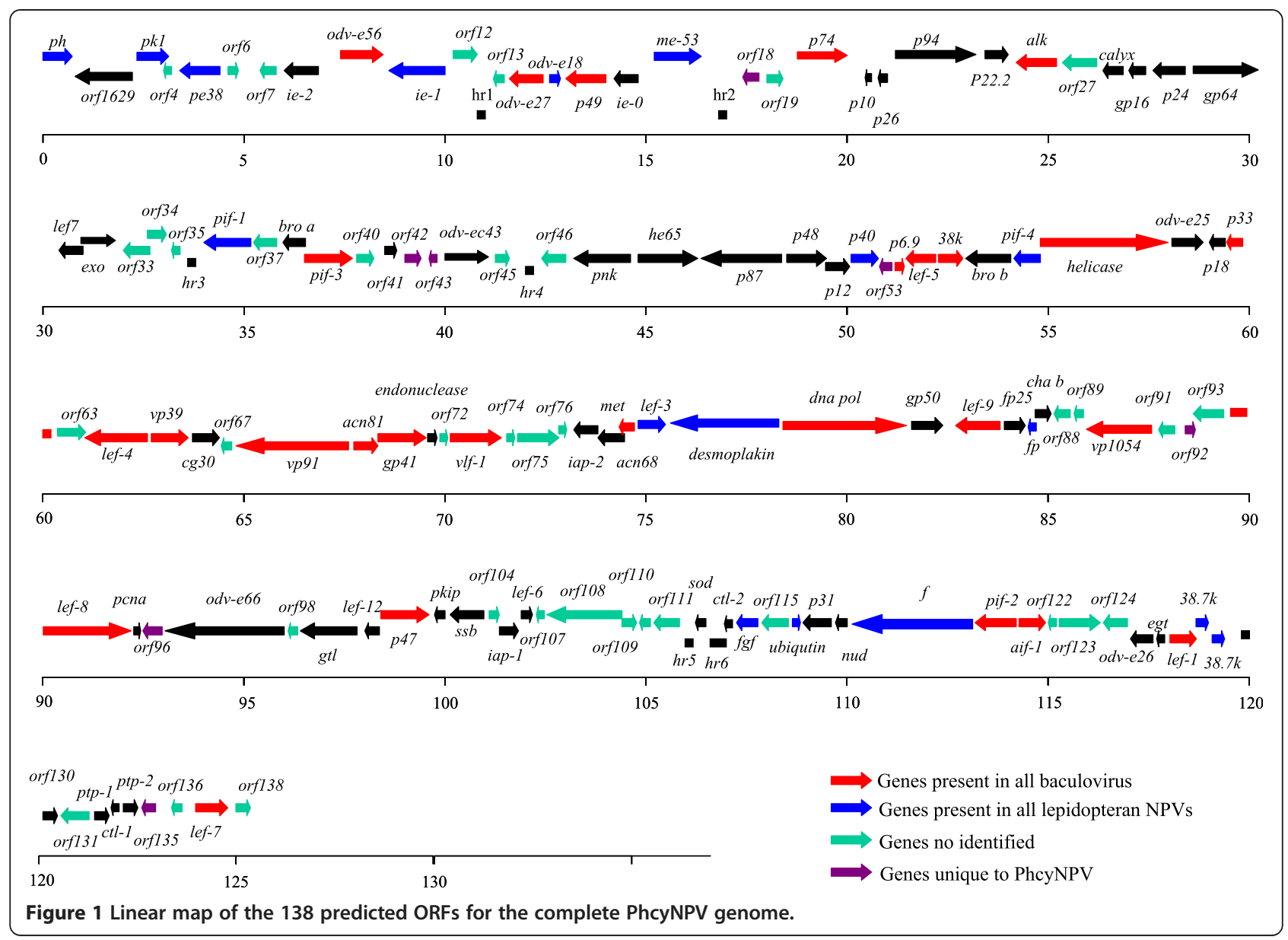

homologs are found in all Groups I, II and most GV genomes, mostly encoding proteins of 400 to 500 aa. The function of EGT is to block molting and pupation in infected larvae by catalyzing the transfer of glucose from UDP-glucose to ecdysteroids, thereby inactivating these insect molting hormones [16,17]. Deletion of the egt gene from the genome of AcMNPV caused the virus to kill the insects more quickly [18]. The day after $P$. cynthia ricini larvae were infected with PhcyNPV or AnpeNPV by per os inoculation, nearly one-third of the insects died. This might have been caused by egt mutation. The observation requires further investigation.

\section{Predicting cellular location of the PhcyNPV genes}

Viral proteins that contain a signal peptide are usually involved in viral particle packaging or interaction with hosts. To determine which genes might be related to host interaction, we predicted the presence of a signal peptide in the proteins encoded by all the ORFs of PhcyNPV. Twenty-three gene products of PhcyNPV were identified that might be located in the endoplasmic reticulum (ER). Most of the proteins were found to function in viral packaging and interaction with hosts
(Table 1). Among the 23 genes, six are unique to the three NPVs compared in this paper, and one unknown gene is unique to PhcyNPV. The fusion $(f)$ gene was the only gene whose protein product showed a low probability of being located in the ER (prediction value 0.33). We analyzed the $f$ homolog genes from other baculoviruses and they appear to be divided into two groups: those with high identities (prediction value 0.7, such as AngeNPV, GenBank: YP_803416) and those with low possibilities (prediction value 0.3, AcMNPV, GenBank: NP_054052) (data not shown).

We also identified 19 gene products that might be located in the mitochondria (Table 2). To date, few baculovirus genes have been found to interact with the mitochondria, an important organ involved in energy metabolism and apoptosis. Among the 19 genes, 11 genes have high prediction values (more than 0.5 ), and eight genes have low prediction values (less than 0.5). Interestingly, eight genes are unique to the three compared NPVs; five of them are unique to PhcyNPV.

Of the eight unique predicted genes of PhcyNPV, five might be related to energy metabolism and/or apoptosis, and one is located in ER. These data imply that the unique 


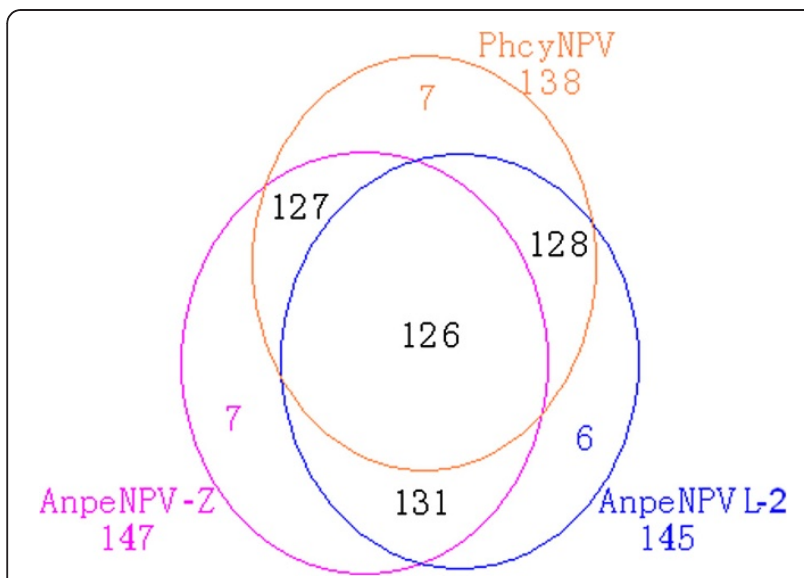

Figure 2 Comparison of the genomes of three types of baculoviruses, PhcyNPV, AnpeNPV-L2 and AnpeNPV-Z. The different circles represent the genes of each type of virus. The numbers contained within the overlapping regions indicate the numbers of shared genes, and the numbers within the circles, but outside the overlapping regions, indicate the number of unique genes in each genome.

genes of baculovirus mostly determine their interaction with individual insect breeds.

\section{The genes related to oral infectivity}

A previous report showed that the cross-infectivity characteristics of PhcyNPV and AnpeNPV were different. AnpeNPV caused $57 \%$ mortality in larvae of $P$. cynthia rici, whereas PcrNPV did not kill the larvae of $A$. pernyi [19]. Hence, the six genes (pif 0 to 5) required for infectivity via the oral route were analyzed. Most of them are very similar between PhcyNPV and the two AnpeNPV isolates, except for PIF-3, which in PhcyNPV has a 59aa extra amino acid sequence in the $\mathrm{N}$-terminus.

\section{Discussion}

Improvements in sequencing technology have made the analysis of the genome sequence of a baculovirus easier. However, purifying a type of baculovirus is not easy if a permissive insect cell line for the viral infection has not been established. At present, no permissive line for PhcyNPV is available. We collected budded virus (BV) of PhcyNPV from larvae infected by oral inoculation, and attempted to infect some common insect cell lines, such as BmN, Sf9, Tn5, and Spli cells; however, no symptoms of infection were observed. We then inoculated the $P$. cynthia rici pupae using the BV obtained, and seeded the infected hemocyte cells on the plate containing insect medium with soft agar gel (0.5\%). After 3-5 days of culture, when polyhedra were observed in the cells, the gel was aspirated and injected into healthy pupae. After several repeats, we obtained purified PhcyNPV. The DNA was digested with restriction endonucleases and showed clear bands (Figure 3).

We observed that PhcyNPV was not infectious to $A$. pernyi by oral inoculation. This might involve the oral infection factors, including $p 74 /$ pif-0 [20], pif-1 [21], pif-2 [22], pif-3 [23], pif-4/19 k/odv-e28 [24], and pif-5/ $o d v-e 56[25,26]$. These six genes are all structural components of occlusion derived viruses (ODVs). P74 mediates the specific binding of ODVs to primary cellular targets in the midgut epithelia [20], while pif-3 appears to mediate another crucial, but as yet unidentified, event during primary infection [23]. The proteins encoded by pif- 4 and pif- 5 have essential roles in the per os infection route [24-26]. Most of these genes are highly similar to those of AnpeNPV, except for PIF-3 of PhcyNPV, which has an extra 59-aa N-terminal structure. However, no homologous structure was found in other viruses or species. The PIF-3 proteins from other baculoviruses are all about 200 aa, and show low levels of sequence similarity to each other. Interestingly, the Cterminal structure of PhcyNPV PIF-3 is similar to that

Table 1 PhcyNPV ORFs with a predicted subcellular location in the ER (endoplasmic reticulum)

\begin{tabular}{|c|c|c|c|}
\hline Gene number & ORF (aa) & Gene name & Prediction value \\
\hline PhcyNPV012 & 95 & chitin-binding protein 1 & 0.99 \\
\hline PhcyNPV028 & 102 & gp16 & 0.99 \\
\hline PhcyNPV030 & 509 & gp64 & 0.94 \\
\hline PhcyNPV036 & 531 & pif-1 & 0.99 \\
\hline PhcyNPV053 & 99 & Phcy-ORF053 & 0.58 \\
\hline PhcyNPV058 & 172 & pif-4/19 k/odv-e28 & 0.99 \\
\hline PhcyNPV060 & 228 & odv-e25 & 0.99 \\
\hline PhcyNPV061 & 158 & p18 & 0.90 \\
\hline PhcyNPV063 & 191 & Anpe-ORF064 & 0.99 \\
\hline PhcyNPV074 & 84 & Anpe-ORF077 & 0.99 \\
\hline PhcyNPV083 & 362 & gp50 spindlin & 0.99 \\
\hline PhcyNPV088 & 167 & Anpe-ORF093 & 0.90 \\
\hline PhcyNPV091 & 146 & Anpe-ORF098 & 0.61 \\
\hline PhcyNPV097 & 680 & odv-e66 & 0.99 \\
\hline PhcyNPV113 & 63 & $\mathrm{ctl} 2$ & 0.83 \\
\hline PhcyNPV114 & 185 & fgf & 0.92 \\
\hline PhcyNPV115 & 270 & Anpe-ORF123 & 0.99 \\
\hline PhcyNPV119 & 654 & F protein & $0.33 *$ \\
\hline PhcyNPV120 & 382 & pif-2 & 0.99 \\
\hline PhcyNPV121 & 324 & arif-1 & 0.99 \\
\hline PhcyNPV126 & 87 & egt & 0.99 \\
\hline PhcyNPV131 & 336 & Anpe-ORF139 & 0.91 \\
\hline PhcyNPV133 & 53 & ctl 1 & 0.99 \\
\hline
\end{tabular}

*Low probability of an ER location; bold face indicates an ORF found only in the PhcyNPV genome. 
Table 2 PhcyNPV ORFs with a predicted mitochondrial location

\begin{tabular}{lllc}
\hline Gene number & ORF (aa) & Gene name & Prediction value \\
\hline PhcyNPV006 & 69 & Anpe-ORF006 & 0.60 \\
PhcyNPV018 & 82 & Phcy-ORF018 & $0.41^{*}$ \\
PhcyNPV025 & 465 & alkaline exonuclease & $0.24^{*}$ \\
PhcyNPV032 & 285 & EXO III v-trex & 0.92 \\
PhcyNPV039 & 264 & pif-3 & 0.67 \\
PhcyNPV042 & 109 & Phcy-ORF042 & $0.21^{*}$ \\
PhcyNPV043 & 68 & Phcy-ORF043 & 0.83 \\
PhcyNPV050 & 387 & P48 & $0.22^{*}$ \\
PhcyNPV054 & 79 & p 6.9 & 0.83 \\
PhcyNPV056 & 313 & 38 K & 0.73 \\
PhcyNPV062 & 253 & P33 & $0.38^{*}$ \\
PhcyNPV069 & 214 & AcN81 & $0.25 *$ \\
PhcyNPV071 & 98 & endonuclease & $0.45^{*}$ \\
PhcyNPV078 & 264 & methyl transferase & 0.56 \\
PhcyNPV090 & 376 & vp1054 & $0.37^{*}$ \\
PhcyNPV092 & 117 & Phcy-ORF092 & 0.68 \\
PhcyNPV096 & 223 & Phcy-ORF096 & 0.54 \\
PhcyNPV098 & 157 & Anpe-ORF105 & 0.68 \\
PhcyNPV136 & 149 & Anpe-ORF143 & 0.70 \\
\hline
\end{tabular}

*Low probability of a mitochondrial location; bold face indicates an ORF found only in the PhcyNPV genome.

of a membrane-spanning Ca-ATPase from the fungus Spathaspora passalidarum (GenBank: EGW31824), and an $\mathrm{ABC}$ transporter exported protein from the bacterium Pseudomonas sp. R81 (GenBank: ZP_11190693). These proteins are associated with the cell membrane.

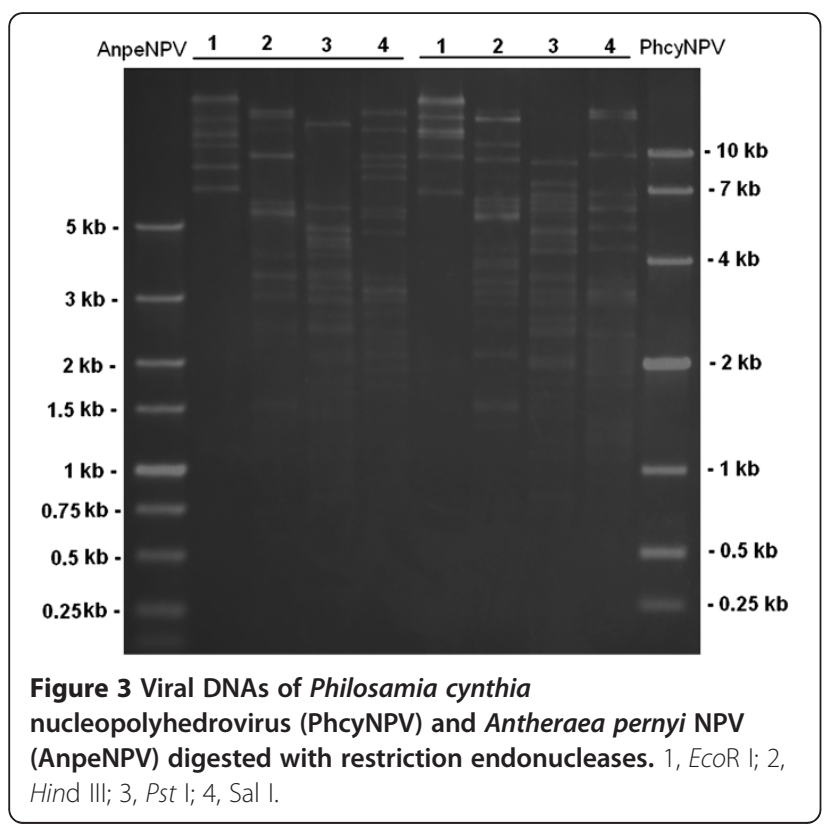

These observations imply that baculovirus PIF-3 might be related to the viral trans-membrane process.

The hrf1 gene from LdMNPV expands the host range of AcMNPV both in vitro and in vivo, allowing it to infect non-permissive hosts $[27,28]$. The only baculovirus homo$\log$ of this gene is found in OpMNPV. Two conotoxin-like ( $c t l$ ) genes, $c t l 1$ and $c t l 2$, are also found in both of these genomes. Other baculoviruses encode one or the other, but only OpMNPV and LdMNPV encode both. A report indicated that there is a clear phylogenetic link between hrfland the presence of both $c t l$ genes [29]. We also found the two $c t l$ genes in the genomes of PhcyNPV and AnpeNPV. However, the $h r f 1$ gene was missing. It seems that functions of $c t l 1$ and $c t l 2$ are distinct when appearing with $h r f 1$.

\section{Conclusions}

Most of the genes in PhcyNPV were similar to the two AnpeNPV isolates, including the direction of expression of the ORFs. Only a few genes were missing in PhcyNPV. These data suggest that PhcyNPV and AnpeNPV might be variants with each other, and the difference of crossinfection might be caused by gene mutations.

\section{Methods \\ Viruses}

The Institute of Guangxi Sericultural Research and Development in South China provided the occlusion bodies (OBs) of PhcyNPV. The AnpeNPV was kindly provided by Professor Qin Li of Shenyang Agricultural University in north China.

\section{Viral DNA preparation and sequencing}

The procedures for isolating $\mathrm{OBs}$ and preparing viral DNA were as described by Cheng et al., 2005 [30]. In brief, the OBs were purified by density gradient centrifugation, and were dissociated with a lysis buffer containing 0.1 $\mathrm{mol} / \mathrm{L} \mathrm{Na} \mathrm{CO}_{3}$ and $0.15 \mathrm{~mol} / \mathrm{L} \mathrm{NaCl}$ on ice for $30 \mathrm{~min}$. After that, $0.5 \%$ SDS and proteinase $\mathrm{K}(50 \mathrm{mg} / \mathrm{mL})$ were added and incubated at $37^{\circ} \mathrm{C}$ for $4 \mathrm{~h}$. The digested solution was progressively extracted with phenol and chloroform mixtures. DNA was precipitated with $70 \%$ ethanol. The DNA was dried and dissolved in $2.0 \mathrm{mmol} / \mathrm{L}$ Tris $(\mathrm{pH}$ 8.0). The quantity and quality of the isolated DNA were determined spectrophotometrically and by electrophoresis on $0.7 \%$ agarose gel. A DNA fragment library of PhcyNPV was constructed using the shotgun method described by Zhu et al. [31]. All clones were sequenced and the fulllength sequence was constructed by the Chinese National Human Genome Center at Beijing.

\section{Sequence analysis}

ORFs in the PhcyNPV genome were identified using ORF finder (http://www.ncbi.nlm.nih.gov/gorf/gorf.html). All 
BLAST searches were done through the National Center for Biotechnology Information (NCBI) websites. The signal peptide data were downloaded from http://www. cbs.dtu.dk/services/SignalP/ using the software Prodotar v.1.03.

\section{Additional file}

Additional file 1: Table S1. Predicted ORFs in the PhcyNPV genome, comparing to the closely related AnpeNPV.

\section{Competing interests}

There are no competing interests, including financial competing interests, in this paper.

\section{Author' contributions}

$A X$ and WW conceived the project and drafted the final manuscript. $\mathrm{HQ}$ and YZ carried out the molecular genetic studies. PS and XG participated in its design and coordination and helped to draft the manuscript. $\mathrm{HQ}$ and KG participated in the design of the study. HQ, YW and SZ participated in the sequence alignment. All authors read and approve the final manuscript.

\section{Acknowledgements}

This work was supported by a grant from the China Agriculture Research System (Sericulture industry, CARS-22-ZJ0101).

\section{Author details}

${ }^{1}$ Jiangsu University of Science and Technology, Sibaidu Road 1, Zhenjiang 212018, China. ${ }^{2}$ Jiangsu University, Xuefu Road 301, Zhenjiang 212013, China. ${ }^{3}$ The Sericultural Research Institute, Chinese Academy of Agricultural Sciences, Jiangsu University of Science and Technology, Sibaidu Road 1, Zhenjiang 212018, China.

Received: 17 October 2012 Accepted: 31 January 2013 Published: 20 February 2013

\section{References}

1. van Oers MM, Vlak JM: Baculovirus Genomics. Current Drug Targets 2007, 8:1051-1068.

2. Herniou EA, Olszewski JA, O'Reilly DR, Cory JS: Ancient Coevolution of Baculoviruses and Their Insect Hosts. J Virol 2004, 78:3244-3251.

3. Miele SAB, Garavaglia MJ, Belaich MN, Ghiringhelli PD: Baculovirus: molecular insights on their diversity and conservation. Int J Evol Biol 2011, 2011:1-15.

4. Zhang G: Research, development and application of Heliothis viral pesticide in China. Resour Environ in the Yangtze Valley 1994, 3:1-6.

5. Kost TA, Condreay JP: Recombinant baculoviruses as expression vectors for insect and mammalian cells. Curr Opin Biotechnol 1999, 10:428-433.

6. Kost TA, Condreay JP, Jarvis DL: Baculovirus as versatile vectors for protein expression in insect and mammalian cells. Nat Biotechnol 2005, 23:567-575.

7. Liu YQ, Qin L, Li YP, Wang H, Xia RX, Qi YH, Li XS, Lu C, Xiang ZH: Comparative genetic diversity and genetic structure of three Chinese silkworm species Bombyx mori L. (Lepidoptera: Bombycidae), Antheraea pernyi Guérin-Meneville and Samia cynthia ricini Donovan (Lepidoptera: Saturniidae). Neotrop Entomol 2010, 39:967-976.

8. Guo $Y$, Shen $Y H$, Sun W, Kishino $H$, Xiang ZH, Zhang Z: Nucleotide diversity and selection signature in the domesticated silkworm, Bombyx mori, and wild silkworm, Bombyx mandarina. J Insect Sci 2011, 11:1-16.

9. Woo SD, Roh JY, Choi JY, Jin BR: Propagation of Bombyx mori Nucleopolyhedrovirus in nonpermissive insect cell lines. J Microbio/ 2007, 45:133-138.

10. Fan $Q$, Li S, Wang L, Zhang B, Ye B, Zhao Z, Cui L: The genome sequence of the multinucleocapsid nucleopolyhedrovirus of the Chinese oak silkworm Antheraea pernyi. Virology 2007, 366:304-315.

11. Nie ZM, Zhang ZF, Wang D, He PA, Jiang CY, Song L, Chen F, XU J, Yang L, Yu LL, Chen J, Lv ZB, Lu JJ, Wu XF, Zhang YZ: Complete sequence and organization of Antheraea pernyi nucleopolyhedrovirus, a dr-rich baculovirus. BMC Genomics 2007, 8:248-261
12. Maeda S, Kamita SG, Kondo A: Host range expansion of Autographa californica nuclear polyhedrosis virus (NPV) following recombination of a 0.6-kilobase-pair DNA fragment originating from Bombyx mori (NPV). J Virol 1993, 67:6234-6238.

13. Gomi S, Majima K, Maeda S: Sequence analysis of the genome of Bombyx mori nucleopolyhedrovirus. J Gen Virol 1999, 80:1323-1337.

14. Xu YP, Ye ZP, Niu CY, Bao YY, Wang WB, Shen WD, Zhang CX: Comparative Analysis of the Genomes of Bombyx mandarina and Bombyx mori Nucleopolyhedroviruses. J Microbiol 2010, 48:102-110.

15. Clem RJ, Robson M, Miller LK: Influence of infection route on the infectivity of baculovirus mutants lacking the apoptosis-inhibiting gene p35 and the adjacent gene p94. J Virol 1994, 8:6759-6762.

16. O'Reilly DR, Miller LK: A baculovirus blocks insect molting by producing ecdysteroid UDPglucosyl transferase. Science 1989, 245:1110-1112.

17. O'Reilly DR, Miller LK: Regulation of expression of a baculovirus ecdysteroid UD Pglucosyltransferase gene. J Virol 1990, 64:1321-1328.

18. Flipsen JT, Mans RM, Kleefsman AW, Knebel-Mörsdorf D, Vlak JM: Deletion of the baculovirus ecdysteroid UDP-glucosyltransferase gene induces early degeneration of Malpighian tubules in infected insects. J Virol 1995, 69:4529-4532

19. Chen Y, Li B, Shen W, Zhu S, Wu Y, Wang W: Analysis of oral infection and helicase gene of the nucleopolyhedroviruses isolated from Philosamia cynthia ricini and Antheraea pernyi. Biocontrol Sci Technol 2008, 18:9967-9973.

20. Mikhailov V: Characterization of $3^{\prime}->5^{\prime}$ exonuclease associated with DNA polymerase of silkworm nuclear polyhedrosis virus. Nucleic Acids Res 1986, 14:3841-3857.

21. McDougal W, Guarino LA: Autographa californica nuclear polyhedrosis virus DNA polymerase: measurements of processivity and strand displacement. J Virol 1999, 73:4908-4918.

22. Hang X, Guarino LA: Purification of Autographa californica nucleopolyhedrovirus DNA polymerase from infected insect cells. J Gen Virol 1999, 80:2519-2526.

23. Vanarsdall AL, Okano K, Rohrmann GF: Characterization of the replication of a baculovirus mutant lacking the DNA polymerase gene. Virology 2005, 331:175-180.

24. Fang M, Nie $Y$, Harris S, Erlandson MA, Theilmann DA: Autographa californica multiple nucleopolyhedrovirus core gene ac96 encodes a per os infectivity factor (pif-4). J Virol 2009, 83:12569-12578.

25. Braunagel SC, Elton DM, Ma H, Summers MD: Identification and analysis of an Autographa californica nuclear polyhedrosis virus structural protein of the occlusion-derived virus envelope: ODV-E56. Virology 1996, 217:97-110.

26. Sparks WO, Harrison RL, Bonning BC: Autographa californica multiple nucleopolyhedrovirus ODV-E56 is a per os infectivity factor, but is not essential for binding and fusion of occlusion-derived virus to the host midgut. Virology 2011, 409:69-76.

27. Chen CJ, Quentin ME, Brennan LA, Kukel C, Thiem SM: Lymantria dispar nucleopolyhedrovirus hrf- 1 expands the larval host range of Autographa californica nucleopolyhedrovirus. J Virol 1998, 72:2526-2531.

28. Thiem SM, Du XL, Quentin ME, Berner MM: Identification of a baculovirus gene that promotes Autographa californica nuclear polyhedrosis virus replication in a nonpermissive insect cell line. J Virol 1996, 70:2221-2229.

29. Herniou EA, Olszewski JA, Cory JS, O'Reilly DR: The genome sequence and evolution of baculoviruses. Annu Rev Entomol 2003, 48:211-234.

30. Cheng XW, Carnerb GR, Langec M, Jehlec JA, Arif BM: Biological and Molecular Characterization of a Multicapsid Nucleopolyhedrovirus from Thysanoplusia orichalcea (L.) (Lepidoptera: Noctuidae). J Invertebral Pathology 2005, 88:126-135.

31. Zhu SY, Yi JP, Shen WD, Wang LQ, He HG, Wang Y, Li B, Wang WB: Genomic sequence, organization and characteristics of a new nucleopolyhedrovirus isolated from Clanis bilineata larva. BMC Genomics 2009, 10:91-99.

doi:10.1186/1471-2164-14-115

Cite this article as: Qian et al:: Analysis of the genomic sequence of Philosamia cynthia nucleopolyhedrin virus and comparison with Antheraea pernyi nucleopolyhedrin virus. BMC Genomics 2013 14:115. 